\title{
A novel approach to reverse proteinopathies
}

BRD4780 ...
releases
MUC $1 \mathrm{fs}$ from
the early
secretory
pathway,
enabling transit
of MUC1fs into
lysosomes to
be degraded

Autosomal dominant tubulointerstitial kidney disease-MUC1 (ADTKD$M U C 1)$ is a slowly progressive disease, for which there are no therapies and the pathogenetic mechanism is unknown. Writing in Cell, Dvela-Levitt et al. report that ADTKD-MUC1 is a toxic proteinopathy and identify a small molecule that clears the accumulated toxic protein by modifying its intracellular trafficking.

Mucin 1 (MUC1) is a transmembrane mucin expressed in epithelial cells. In patients with ADTKD-MUC1, frameshift (fs) mutations in $M U C 1$ produce a truncated protein, MUC1fs.

To dissect how MUC1fs causes ADTKD, the authors first examined the subcellular localization of wild-type MUC1 (MUC1wt) and MUC1fs in kidney biopsy samples from a patient heterozygous for the MUC1 frameshift mutation. MUC1wt was present in the apical membrane in tubule and collecting duct cells, whereas MUC1fs accumulated

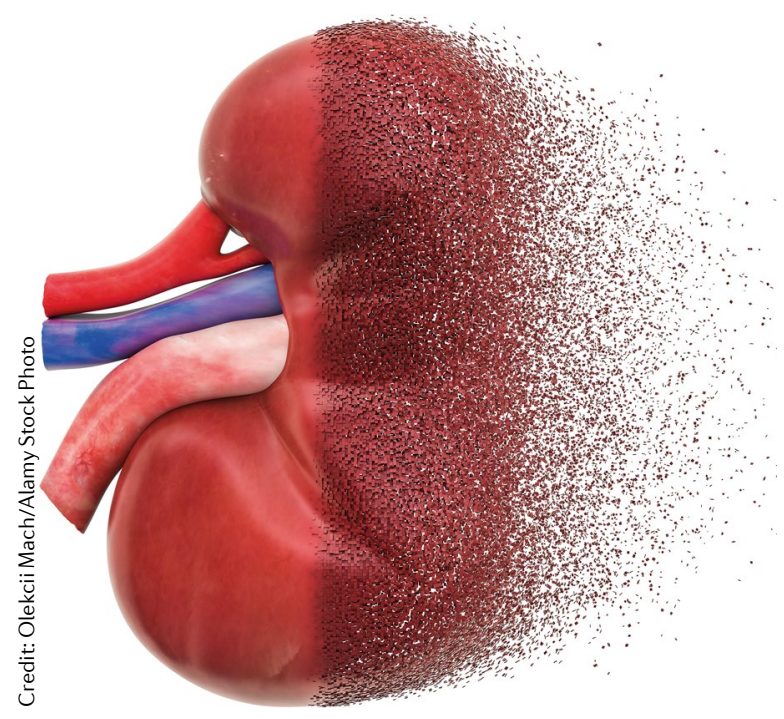

intracellularly, suggesting that MUC1fs is retained in the secretory pathway and does not transit to the plasma membrane. These results were confirmed in heterozygous knock-in mice expressing a human MUC1fs allele (+/fs mice), in kidney organoids generated from patient induced pluripotent stem cells and in a patient-derived immortalized tubular epithelial cell line (+/MUC1fs cells). Importantly, $+/$ fs mice developed a similar tubulointerstitial pathology to that in patients with ADTKD-MUC1.

Immunofluorescence analysis with a panel of secretory pathway markers revealed that MUC1fs accumulates in the cis-Golgi and in vesicles marked with the cargo receptor TMED9, which is involved in Golgi-endoplasmic reticulum (ER) retrograde transport.

Next, the authors investigated whether MUC1fs accumulation triggered the unfolded protein response (UPR), which attempts to maintain proteostasis in the face of ER stress. Analysis of RNA sequencing data and follow-up studies probing the UPR at the protein level indicated that the ATF6 branch of the UPR was upregulated in +/MUC1fs cells compared with control $(+/+)$ cells. Furthermore, ATF6 inhibition exacerbated MUC1fs accumulation in +/MUC1fs cells and led to increased apoptosis compared with control cells. In fact, treatment with the general ER stressor thapsigargin increased apoptosis in +/MUC1fs cells in vitro and in vivo. Thus, MUC1fs accumulation causes a toxic proteinopathy, and activation of ATF6 attempts to protect tubular epithelial cells from this toxicity.

The authors screened a drug repurposing library of 3,713 drugs to identify compounds that could relieve the block in MUC1fs intracellular transport (that is, reduce MUC1fs levels by $>30 \%$, without cellular toxicity). Additional screens using a wider range of doses and eliminating compounds that reduced MUC1wt levels or MUC1fs mRNA levels, or did not rescue thapsigargininduced apoptosis in +/MUC1fs cells, whittled down the 203 hits to a single compound, BRD4780.

Administration of BRD4780 to +/fs mice reduced MUC1fs but not MUC1wt levels in the kidneys, and similar results were obtained in kidney organoids derived from patients. In a time course, BRD4780 promoted transit of MUC1fs from the early secretory pathway to endosomes and lysosomes, but only in the presence of a functional secretory pathway. In fact, TMED9 levels were increased in +/MUC1fs cells, and TMED9 deletion phenocopied the effect of BRD4780 on MUC1fs accumulation. Mechanistically, the authors provided evidence suggesting that BRD4780 binds directly to TMED9, which releases MUC1fs from the early secretory pathway, enabling transit of MUC1fs into lysosomes to be degraded.

This ability of BRD4780 to promote removal of misfolded proteins that accumulate in the secretory pathway extends to other proteinopathies, both of the kidneys and other organs. For example, in cell lines, BRD4780 treatment reduced the levels of mutant uromodulin, which causes ADTKD-UMOD, and mutant rhodopsin, which causes retinitis pigmentosa. Thus, BRD4780 might have wider therapeutic potential in treating toxic proteinopathies.

This study identifies a novel approach to clear toxic protein accumulations in various, currently untreatable proteinopathies, and BRD4780 represents a valuable therapeutic lead.

Grant Otto

ORIGINAL ARTICLE Dvela-Levitt, M. et al. Small molecule targets TMED9 and promotes lysosomal degradation to reverse proteinopathy. Cell 178, 1-15 (2019) 